\title{
A Universal Data Access and Protocol Integration Mechanism for Smart Home
}

\author{
Pengfei Shao, Member, IACSIT
}

\begin{abstract}
With the lack of standardized or completely missing communication interfaces in home electronics, there is no perfect solution to address every aspect in smart homes based on existing protocols and technologies. In addition, the central control unit (CCU) of smart home system working point-to-point between the multiple application interfaces and the underlying hardware interfaces leads to its complicated architecture and unpleasant performance. A flexible data access and protocol integration mechanism is required. The current paper offers a universal, comprehensive data access and protocol integration mechanism for a smart home. The universal mechanism works as a middleware adapter with unified agreements of the communication interfaces and protocols, offers an abstraction of the application level from the hardware specific and decoupling the hardware interface modules from the application level. Further abstraction for the application interfaces and the underlying hardware interfaces are executed based on adaption layer to provide unified interfaces for more flexible user applications and hardware protocol integration. This new universal mechanism fundamentally changes the architecture of the smart home and in some way meets the practical requirement of smart homes more flexible and desirable.
\end{abstract}

Index Terms - Smart home, central control unit, universal mechanism.

\section{INTRODUCTION}

With the development of economy and science technology, smart homes, as an important part in the direction of wisdom city development are going nearer and nearer to people's everyday lives [1].

Smart homes have their own intelligent control systems. The home control network infrastructure is the basic skeleton for smart home construction in that a smart home unifies home appliances. In recent years, various organizations focusing on home network were formed, and each proposed its own recommendations and standards. However, these smart home relevant technologies are not compatible with the interoperability. The biggest challenge smart home researchers face is the lack of standardized or completely missing communication interfaces in home electronics [2].

\section{NETWORKING TECHNOLOGIES AND STANDARDS FOR SMART HOME CONTROL}

Among the emerging technologies popular in the domain

Manuscript received April 12, 2012; revised May 31, 2012.

P. Shao is with the Faculty of Electronic and Informatin Engineering, Zhejiang Wanli University, 315100 QianHu South Road No.8, Ningbo, Zhejiang, P. R. China (e-mail: cbb_bb@126.com) of smart homes, home fieldbus technologies and short-range low-rate wireless network technologies have attracted more attention from researchers and home appliance manufacturers and proved themselves to be the de facto standards and deployment specifications[3]. According to different transmission mediums, the home fieldbus technologies also can be categorized into three types respectively:

- PLC technologies [4], directly transmit data over power lines, benefiting from the availability and the quantity of electrical outlets in a house. The mainstream protocols in PLC (Power Line Communication) technology are X-10, INSTEON, HomePlug, CEBus, LonWorks and PLC-BUS. The CEBus and LonWorks also support communications over twisted pair, coaxial cable, wireless and other mediums.

- HBS, Japanese home bus system standard, using twisted pair as transmission medium.

- EIB, European install bus standard, supporting communications over twisted pair, coaxial cable, wireless and other mediums

The representative protocols of short-range low-rate wireless network technologies include infrared, wireless $\mathrm{Rf}$, Bluetooth, 802.15.4/ZigBee and Z-wave [5] [6].

In home automation, the main advantages of the PLC technologies superior to other alternatives is the availability of power line outlets in each room for a house, which avoids the costs of additional wiring in most residences and the convenience of the promisingly seamless communication with utilities via power line[7]. X-10 is the most conventional standard in home automation due to its user-friendliness and simplicity in installation, but the drawbacks are obvious: the reliability in data transmission (i.e. data signal via power line is considered as noise and filtered out) and the lack of security mechanism due to the limitation of frame length. The comparably high price of LonWorks overshadows its excellent performance in the sense that the technology is initially oriented towards building automation.

Despite the fact that the short-range low-rate wireless technologies have features in common with the PLC technologies in terms of installation and cost, there are also other issues to be taken into consideration and to be addressed [8]. First of all, they possibly operate on the same ISM frequency band which gives rise to the overload resulting from a high-degree of mutual interference with each other, especially in the case that a WLAN is deployed in house. Secondly, signal attenuation, shadow fading as well as multipath effect in the wireless environment deteriorate the quality of data transmission. In terms of network security, it is tricky to completely achieve encryption and authentication 
in the self-organized wireless network due to the dynamics of the network topology and the constraint in computational power and resources for mobile nodes. In addition, those technologies also show vulnerability to malicious wireless attacks such as jamming, forging of random collision frame, etc.

In a nutshell, each of the networking techniques widely adopted in the field of home appliance control has its strengths and weakness. Lack of standardized or completely missing communication interfaces among these techniques is the main obstacle to the practical home control network. There is no perfect solution to address every aspect in smart homes based on either PLC technologies or short-range wireless network technologies.

\section{POINT-TO-POINT DATA ACCESS}

The CCU is a fundamental part and control heart of a universal smart home model, allowing the integration of different devices and applications into a synchronized environment. All devices are connected to it by either wireless or wired connection. The CCU controls devices and equipment, manages preprogrammed scenarios, and communicates with external resources. CCU monitors operate and control all smart-home components by checking status and sending commands.

In a general-purpose smart home system, the CCU at least comprises communication interfaces as follows:

- User Interface (UI): provides the user with access to some or all of the integrated devices and appliances in a home.

- Devices and Equipment Interface: A set of hierarchical commands, functions or accessible attributes for controlling and operating each integrated device. Any device must comply with at least one of the standard communication protocols.

- Interfaces for Communication Protocols. Such as interfaces for X-10, LonWorks, Homeplug, PLC-BUS, ZigBee, Infrared, BlueTooth, and others.

- Interfaces for AI (Artificial Intelligence) [9]: allows the CCU to draw upon the prediction when needed. The AI inference mechanism reacts according to changing conditions, reflecting suitable responses to different scenarios.

- Interfaces for Database. The database is the main storage point for all data in a smart-home environment. Devices, interfaces, commands, and scenarios are all saved in the database.

- Interfaces for Application Services: allows adding software modules, in order to enhance devices operation and inter-device connectivity.

- Net Interface: provides home control system with connection to external environments, such as Ethernet, WLAN.

All of these CCU communication interfaces can be functionally categorized into two types:

- The upper application interfaces: includes UI, devices and equipment interface, AI interface and interfaces for application services.

- The underlying hardware driver interfaces: includes interfaces for communication protocols, DB interface and Net interface etc.

As discussed above, there are many existing network technologies and standards for smart home. But neither home fieldbus technologies nor short-range low-rate wireless technologies can provide a perfect solution for the creation of smart homes because of the lack of universal communication interfaces. This problem can be alleviated by using adapters and converters, however there are limits on what each interface can do and usually some functions remain unavailable. It is in practice more desirable to use multiple network technologies instead of one single network technology. For example, considering the aspect of power conservation, openness of protocol stack, interoperability based on layering and cost-effectiveness, a backbone network of HomePlug C\&C plus ZigBee seems more promising for home appliance control in a smart home [10]. Combining $\mathrm{X}-10$ with ZigBee is more practical when installing networks and devices in older buildings [11]. In such cases, the CCU is required to access multiple hardware networks at the same time. Many point-to-point data accesses and communications occur between application interfaces and hardware driver interfaces of the CCU, shown as Fig.1.

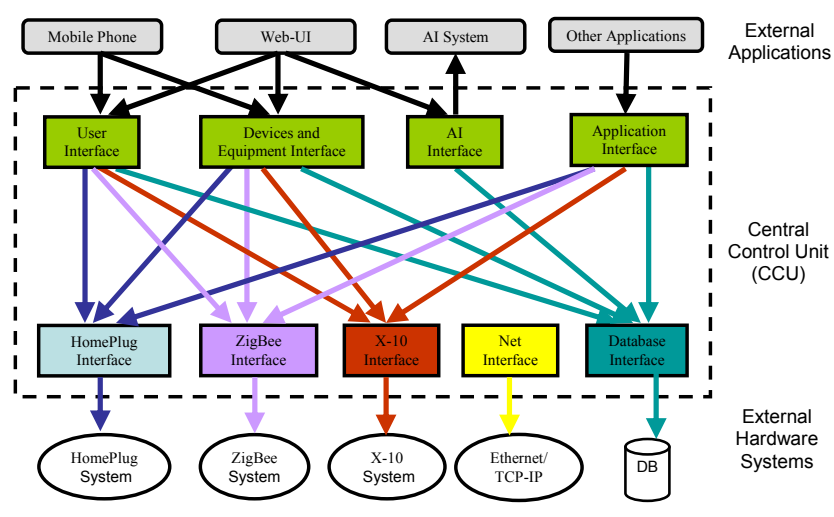

Fig. 1. Point-to-point data access and communication

So working on point-to-point mode, the CCU needs to provide related protocol and hardware interface for the integration or replacement of a new type of external hardware network. Once a new hardware network is integrated or replaced in a smart home, the complexity to access increases linearly between application interfaces and hardware interfaces. This will greatly increase the CCU load and alleviate its performance. In practical applications, some smart home system designs even ask for the CCU to support all the network technologies applicable to the control of home networking. As also can be seen from figure 1, the Net interface only performs a single function to implement interconnected communication with home broadband network or external high-speed network, but not exploit the great potential of remote intelligent supervisor to the smart home via Internet [12], it is closely related to the complexity for the interface access mechanism of current CCU.

Moreover, in a proprietary customized network it might require large modifications to the infrastructure and UIs in order to fully utilize the new device. Even if the protocol and network would support on-the-fly addition of devices the biggest challenge lies in creating dynamic UIs that 
automatically change when changes have been made in the infrastructure [11]. CCU working on a more flexible data access and communication mechanism is required to simplify this operation and configuration.

\section{Proposed Univsrsal Mechanism}

The proposed universal mechanism for inter-interface communications and protocols integration is in a form of middleware adapter. It provides a unified adaption layer for application interface, hardware drivers and protocols, replaces point-to-point data access and communication with bus mode communication mechanism between interfaces, shown as figure 2 . The adaption layer offers an abstraction of the application level from the hardware specific and decoupling the hardware interface modules from the application level. Further abstract for the application interfaces and the underlying hardware interfaces are executed based on the adaption layer to provide unified interfaces for more flexible user applications and hardware protocol integration. This new universal mechanism fundamentally changes the architecture of the smart home and in some way meets the practical requirement in smart homes more flexible and desirable.

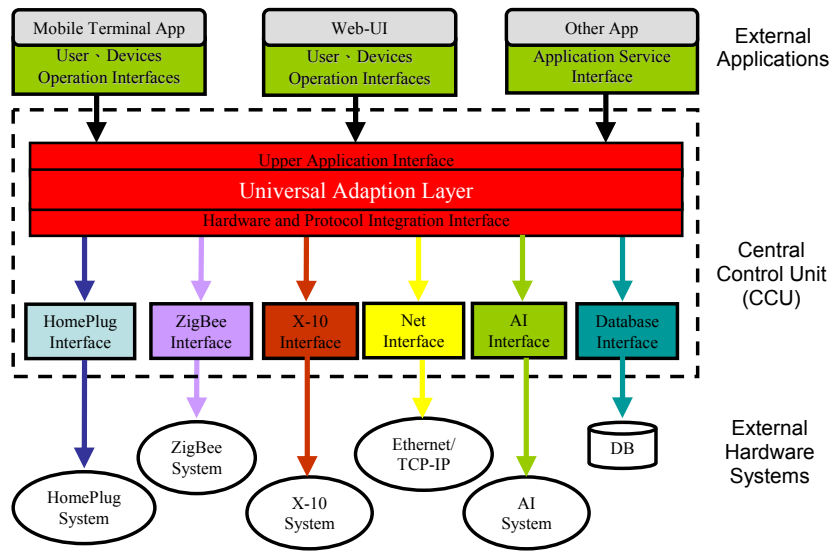

Fig. 2. Universal mechanism based on middleware adapter

\section{A. Universal Adaption Layer}

The middleware adapter mainly deals with data communications both on down link and up link. On the down link, it accepts requirements from home applications via application interfaces and throws them to specific hardware driver or protocol interface instance to acting upon the environment through device controllers. On the up link, it receives sensor status from hardware interfaces and sends them to particular application interfaces for further processing by home appliance. It can thus be seen that the key to design the universal adaption layer is to create binding relations between specific instances of the upper application interfaces and the underlying hardware interfaces, and build the communication mechanism between the internal CCU interfaces. Binding relations can be created by binding a unique identifier of the application interface instance with one concrete underlying hardware driver instance.

The middleware adapter uses a messages mechanism for interacting with hardware interfaces. Smart home-related activities should be regarded as a set of processes and behaviors - rather than a list of separated commands- and implemented as events. The messages mechanism appropriately agrees with this feature. Message queuing service is a loosing coupling distributed application integration form. It classifies messages from different up-to-down bindings of the application interface instances and hardware interface instances, and triggers the blocking internal communications between interfaces. It works asynchronously and supports one-to-more communications. In this design, most of the activation processes are implemented asynchronously. Various mechanisms are adopted to synchronize these asynchronous operations, such as a timeout, waiting for $\mathrm{I} / \mathrm{O}$ completion, value changing, the message board, and etc.

\section{B. Unified Application Interfaces}

In the new architecture, the upper application interfaces are further abstracted based on the universal adaption layer. It provides new application interfaces with permission to access atomic data unit like integer, floating-point data, string and data set. Only several basic communication primitives are opened to do these operations. 'Set' and 'Get' used for data exchange upon application layer. 'Require' and 'Send' used to implement external $\mathrm{I} / \mathrm{O}$ operations through underlying hardware interfaces. Some of current state, ongoing activities, warning messages and relevant information are automatically displayed by 'Indicator', while others appear only upon request. The further abstraction decouples UIs, devices and equipment interfaces and application services interfaces from the CCU. All these application interfaces are now carried out by specific application modules. In other word, each application has its own application interfaces to access the unified application interfaces supported by the universal adaption layer. Fig. 3 shows the application interface definition and configuration for a light control system. By this way, the universal mechanism greatly reduces data access resources and processing costs of the CCU.

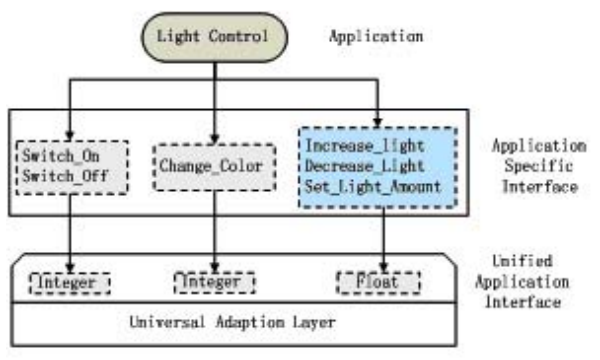

Fig. 3. Application interface abstraction

\section{Unified hardware integration interfaces}

The unified hardware abstraction interfaces based on the universal adaption layer allows easy integration of new hardware devices and communication protocols into the system. Each class of hardware devices or communication protocols supported by the middleware adapter is represented by a hardware interface. The hardware interface is responsible for implementation of the basic I/O operations upon the atomic data unit assigned to it. Further, other 
interfaces (in addition to the application interfaces, such as AI interface, Net interface) can also be abstracted to a virtual underlying hardware interface. This is similar to the virtual file management for external devices in Linux system. In order to exploit its great potential, the Net interface is re-encapsulated as a hardware interface for networking. The new Net interface provides a highly optimized implementation of the networking interface. It corresponds to the presentation and application levels within the OSI model, and functions over different transport layers, but its most compelling use is based on Ethernet TCP/IP sockets. It is fully transparent for application using it with data synchronization between distributed partitions maintained in background. By this way, it may open a desirable solution of remote intelligent supervisor to the smart home via Internet.

A proper event-management control mechanism is very important in a smart home environment. In the new architecture, special event triggers can be configured to perceive the state of the sensors and trigger effectors to act upon the environment among the middleware adapter hardware instances.

\section{CONCLUSION}

The smart home environment is rapidly becoming a reality. However, the lack of standardized or completely missing communication interfaces between the existing home control network technologies results in the CCU must support most of the self-defined home networking standards. Working on point to point mode, once a new hardware network is integrated or replaced in a smart home, the CCU complexity of data access increases linearly between application interfaces and hardware interfaces. This will greatly enhance system load and alleviate system performance. Integration of devices and user interfaces produced by various manufacturers is almost impossible. In order to promote the emergence of a smart-home environment, a universal data access and protocol integration mechanism is provided. It offers an abstraction of the application level from the hardware specific and decoupling the hardware interface modules from the application level. A universal adaption layer is designed to replace point to point data access with bus mode communication mechanism between application interfaces and hardware interfaces. New hardware devices and network can be seamlessly integrated to the system under the new mechanism.

This new universal mechanism fundamentally changes the architecture of the smart home, can substantially lead to higher quality and usability of both devices and user interface and reduce system costs. Based on current available communication standards and infrastructure, the middleware adapter mechanism can easily be applied to any home automation system.

A future smart home control system should contain some kind of adaptability and pro-activity. The next step for this effort will be to implement this mechanism in the context of actual smart environment.

\section{REFERENCES}

[1] V. Ricquebourg, D. Menga, D. Durand, B. Marhic, L. Delahoche, and C. Loge, "The Smart Home Concept: our immediate future," IST IEEE International Conference on E-Learning in Industrial Electronics, pp. 23-28, 2006.

[2] D. Bregman and A. Korman, "A Universal Implementation Model of the Smart Home," International Journal of Smart Home, vol. 3, no. 3, pp. 15-30, 2009

[3] J. Cheng and T. Kunz, "A survey on Smart Home Networking," Carleton University, Systems and Computer Engineering, Technical Report SCE-09-10, September 2009.

[4] Y. J. Lin, H. A. Latchman, M. Lee, and S. Katar, "A power line communication network infrastructure for the smart home," IEEE Wireless Communications, vol. 9, no. 6, pp.104-111, 2002.

[5] B. K. Kim and S. K. Hong, etc, "The Study of Applying Sensor Networks to a Smart Home," Fourth International Conference on Networked Computing and Advanced Information Management, vol. 1, pp. $676-681,2008$.

[6] D. Surie, O. Laguionie, and T. Pederson, "Wireless sensor networking of everyday objects in a smart home environment," International Conference on Intelligent Sensors, Sensor Networks and Information Processing, pp. 189 - 194, 2008.

[7] V. Chunduru and N. Subramanian, "Effects of Power Lines on Performance of Home Control System," International Conference on Power Electronics, Drives and Energy Systems, pp.1 - 6, 2006.

[8] G. Ferrari, P. Medagliani, S. Di Piazza, and M. Martal’o, "Wireless Sensor Networks: Performance Analysis in Indoor Scenarios," EURASIP Journal on Wireless Communications and Networking, 2007.

[9] D. J. Cook and M. Youngblood, etc, "MavHome: An Agent-Based Smart Home," in Proceedings of the First IEEE International Conference on Digital Object Identifier per Com, pp.521 - 524, 2003.

[10] "The ZigBee + HomePlug Smart Energy Marketing Requirements Document", Draft Revision 1.0, March 11, 2009.

[11] [Online].

Available: http://www.homeplug.org/products/ZBHP_SE_MRD_090624.pdf

[12] L. Kaila and J. Mikkonen, etc, "The eHome - a Practical Smart Home Implementation,"

[13] Y. P. Tsou, J. W. Hsieh, C. T. Lin, and C. Y. Chen, "Building a Remote Supervisory Control Network System for Smart Home Applications," IEEE International Conference on Systems, Man and Cybernetics, vol. 3, pp. $1826-1830,2006$.

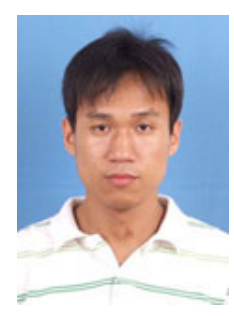

Pengfei Shao (Birth: 3. October 1978, Ningbo, China) 1996.9-2000.7 Undergraduate Studies, Beijing University of Posts and Telecommunications: Communication University of China's best, specialized field: Communication Engineering, completed the relevant degree courses and got bachelor's degree with score the top $10 \%$ in the whole of about 200 graduates. Some network traffic simulations and networking applications development were also done during this period. 2000.9-2003.4

Postgraduate Studies, Beijing University of Posts and Telecommunications, specialized field: Signal and Information Processing, Mentor: Professor ZhenMing Lei, engaged in research of broadband IP networking and embedded system technologies, designed and implemented an integrated access gateway with other five workmates, and finished postgraduate courses and got postgraduate certificate and master's degree.

From September 2000 to April2003, he was a R\&D engineer in

Beijing Kuan Guang Telecom.Hi-Tech Development.Co and majored in embedded systems(pSOS, vxWorks), TCP/IP protocols coding based on freeBSD4.3. From April 2003 to Feb.2005, he worked for Beijing Telecom.Co, implemented and maintained Metropolitan Area Network of Bjtelecom, Beijing core nodes and international Gateway of CHINANET. From Sep.2005 to March 2006, he was a visiting scholar of Applied Science College of Lubeck and Cbb Software Gmbh major in industrial network control, soft middleware, WSN and other technologies. From March 2006 to now, he is a teacher of Zhejiang Wanli University and his current research interests are intelligent network control, the principle, composition and application of the Internet of Things.

Besides, Associate professor Pengfei Shao is a registered member of IACSIT 